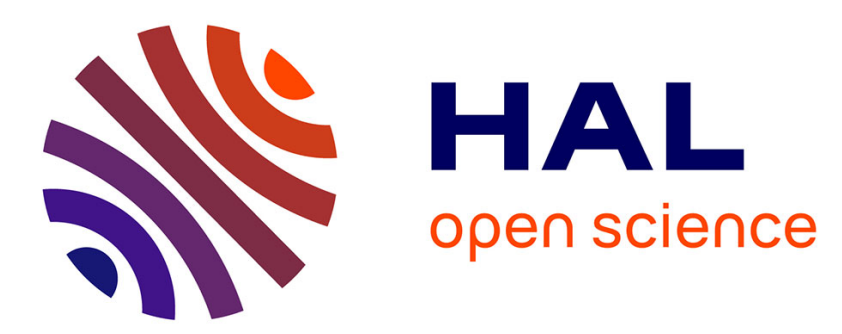

\title{
Classifications ouvrières et confrontation de générations d'ouvriers qualifiés : vers une remise en cause de l'ordre de l'ancienneté?
}

Séverine Misset

\section{- To cite this version:}

Séverine Misset. Classifications ouvrières et confrontation de générations d'ouvriers qualifiés: vers une remise en cause de l'ordre de l'ancienneté?. Sociétés contemporaines, 2013, 2 (90), http://www.cairn.info/revue-societes-contemporaines-2013-2-page-29.htm. 10.3917/soco.090.0029 . halshs-01481165

\section{HAL Id: halshs-01481165 \\ https://shs.hal.science/halshs-01481165}

Submitted on 2 Mar 2017

HAL is a multi-disciplinary open access archive for the deposit and dissemination of scientific research documents, whether they are published or not. The documents may come from teaching and research institutions in France or abroad, or from public or private research centers.
L'archive ouverte pluridisciplinaire HAL, est destinée au dépôt et à la diffusion de documents scientifiques de niveau recherche, publiés ou non, émanant des établissements d'enseignement et de recherche français ou étrangers, des laboratoires publics ou privés. 


\section{Séverine MISSET}

\section{Classifications ouvrières et confrontation de générations d'ouvriers qualifiés : vers une remise en cause de l'ordre de l'ancienneté ?}

À partir d'une enquête de terrain réalisée dans une entreprise de l'industrie métallurgique, cet article analyse comment une confrontation entre générations ouvrières autour de la prise en compte du diplôme et de l'ancienneté, liée à la multiplication des bacheliers professionnels au sein du groupe des ouvriers qualifiés, se voit renouvelée lors de l'introduction d'une nouvelle grille de classification se réclamant d'une "logique compétence ». Si celle-ci semble au premier abord bouleverser les principes de gestion de la population en remettant en cause l'ordre de l'ancienneté, le partage de cet ordre, y compris par l'encadrement de proximité, permet de nuancer ce point de vue.

es effets de la fréquentation prolongée de l'école par une frange des jeunesses ouvrières, suite à la création en 1985 du baccalauréat professionnel, ont été mis en avant pour expliquer les tensions entre générations ouvrières (Beaud et Pialoux, 1999). Dès les premières sessions du diplôme, on note une vague de substitution des bacheliers professionnels aux titulaires de CAP (Hallier et Thiesset, 1991); alors que les promoteurs du baccalauréat professionnel les destinaient initialement à des postes de techniciens, ils sont en effet massivement embauchés sur des postes d'ouvriers qualifiés (Eckert, 1999). Avec la multiplication des titulaires du « bac pro» au sein des entreprises, c'est aussi la figure d'un « nouvel ouvrier », possédant une culture industrielle le prédisposant à l'adhésion aux objectifs économiques de l'entreprise, qui fait irruption dans l'entreprise (Eckert, 1999). Se faisant véhicule des pratiques gestionnaires d'optimisation de la rentabilité et de la productivité, ils peuvent ainsi venir s'opposer aux générations plus anciennes (Beaud, 1996).

Cet article ${ }^{1}$ voudrait insister sur une perspective complémentaire, en étudiant le rôle des pratiques gestionnaires liées aux classifications et la manière dont elles viennent peser sur ces oppositions de générations. L'enquête de terrain, réalisée dans une entreprise de l'industrie métallurgique et centrée sur les ouvriers qualifiés (cf. encadré 1), a en effet été menée à un moment particulier de son histoire : la

1/ Je remercie tout particulièrement les membres du comité de rédaction de Sociétés contemporaines, ainsi que Christian Papinot, pour leurs remarques et suggestions d'amélioration sur des versions antérieures de cet article. 
renégociation des accords de classification de la population ouvrière et la mise en place de nouveaux accords inspirés d'une «logique compétence » (cf. encadré 2).

\section{ENCADRÉ 1 MÉTHODOLOGIE}

L'enquête de terrain a été réalisée entre 2004 et 2007 dans une entreprise de l'industrie métallurgique, en deux temps. Tout d'abord, une recherche collective a été menée auprès d'une trentaine de jeunes ouvriers non qualifiés, embauchés ou intérimaires, à la demande de l'entreprise qui s'interrogeait sur le rapport au travail des jeunes générations ouvrières (Misset, Eme et Hinault, 2004).

Profitant des contacts novés lors de cette étude et du contexte spécifique à l'entreprise (cf. encadré 2), une prolongation de l'enquête a été négociée par la suite ${ }^{2}$, pour se concentrer cette fois sur la population des ouvriers qualifiés, appelés ouvriers professionnels (OP) conformément aux pratiques de la métallurgie. Dans quatre usines contrastées (deux en Île-de-France, une en Lorraine et une en Franche-Comté), 54 entretiens ont été réalisés auprès d'OP salariés de l'entreprise, ainsi qu'une douzaine d'entretiens auprès de responsables hiérarchiques (chefs d'équipe, chefs d'atelier) et de gestionnaires du personnel. Ces données ont été complétées par des observations et des discussions informelles, au siège de l'entreprise et sur les sites étudiés, ainsi que par le recueil de documents (tracts syndicaux, informations chiffrées sur les salariés, documents liés à la négociation en cours, etc.).

Deux générations d'OP, assez nettement divergentes, ont été rencontrées: d'une part, des anciens, souvent âgés de plus de 40 ans et titulaires d'un CAP, et d'autre part, des jeunes, âgés de 20 à 30 ans et ayant moins de dix ans d'ancienneté, qui correspondent donc généralement aux premières cohortes d'embauchés titulaires de "bacs pros ". Ces distinctions en matière de diplôme renvoient en fait à deux générations en termes d'embauche : en effet, l'entreprise a connu un gel des embauches qui n'a cessé qu'à la fin des années 90 .

\section{ENCADRÉ 2}

\section{D'UN ACCORD DE CLASSIFICATION À L'AUTRE}

L'enquête a été effectuée dans un contexte de renégociation des accords de classification et d'évolution professionnelle applicables aux ouvriers. Jusqu'alors, les grilles de classification ${ }^{3}$ en vigueur sont celles signées en juillet 1975 par certains syndicats de la métallurgie et I'UIMM ${ }^{4}$, organisation patronale de cette branche (Pezet, 2000).

2/ Le contexte de réalisation de l'enquête mérite une analyse réflexive détaillée, qui ne peut être menée dans le cadre de cet article. Sur ce point, cf. Misset (2009, p. 39-78).

3/ Une grille de classification permet d'affecter chaque salarié d'un coefficient, qui sert notamment à déterminer sa rémunération minimale, même si d'autres éléments (prime d'insalubrité, prime d'ancienneté, etc.) rentrent également en compte. Mais plus fondamentalement, elle établit des hiérarchies professionnelles et des modalités de carrière (Eyraud et al., 1989).

4/ Union des Industries et Métiers de la Métallurgie (avant le $1^{\text {er }}$ septembre 2001, Union des Industries Métallurgiques et Minières). 
En 1975, prenant acte du "vieillissement » des classifications Parodi-Croizat élaborées après-guerre, les signataires sont parvenus à un accord autour d'une grille dite "à critères classants» (Saglio, 1988, 1991). Dans cette grille, les niveaux scolaires sont mentionnés en référence à des connaissances requises par le poste et non acquises par l'individu (Chatzis, de Coninck et Zarifian, 1995, p. 37). Dans l'esprit des négociateurs de I'UIMM, cette grille prétend donc attribuer des coefficients aux postes de travail et non aux individus qui les occupent. Dans l'entreprise étudiée, un système de " pesée des postes » indique le coefficient théoriquement nécessaire pour occuper un poste. Le poste comporte alors une plage d'évolution de 4 coefficients 13 en dessous de la pesée et celui de la "pesée», qui est le coefficient maximum d'un poste) ${ }^{5}$.

La grille de 1975 est donc censée être un classement des postes et non des individus. Toutefois, l'application de cette grille s'est éloignée de ce principe initial. Tout d'abord, on n'observe pas de remise en cause du coefficient obtenu à un moment donné lors d'une mobilité : dans les faits, la rétrogradation en termes de coefficient est impossible, même lorsque l'individu connaît un déclassement concernant le poste occupé, ce qui marque une différence très importante avec le système initial de "cotation des postes". En outre, le classement minimal octroyé par un niveau de diplôme fonctionne largement comme une garantie : en particulier, la détention d'un baccalauréat professionnel ouvre quasi systématiquement le droit à un accueil au niveau du coefficient 215 , ne serait-ce que parce que les responsables évitent d'embaucher des bacheliers professionnels sur des postes qui ne seraient pas " pesés » à ce niveau-là. De surcroît, l'adéquation entre le coefficient d'un individu et la "pesée du poste " est loin d'être systématique. Ainsi, sur un des sites d'enquête, on a les exemples de Dominique, titulaire d'un coefficient 270 et employé, suite à un reclassement, sur un poste pesé 255 lil dépasse donc le maximum théoriquement possible), ou de Florence, titulaire d'un coefficient 200 et employée sur un poste pesé 255 lelle est donc un coefficient en-dessous du coefficient minimum théoriquement possible).

On peut donc dire qu'à travers ces coefficients, ce sont aussi les individus qui sont classés et que ce classement ne dépend pas forcément du poste effectivement occupé. L'application concrète de l'accord de 1975 semble donc relever d'une logique hybride entre une qualification ramenée au poste de travail et une qualification individuelle.

Ce système a été remis en cause lors de la renégociation, au niveau de l'entreprise, de nouveaux accords se réclamant d'une « logique compétence ». En 2005, un premier accord pour les ouvriers non qualifiés, appelés opérateurs ou APF (agents professionnels de fabrication) dans l'entreprise étudiée, a été signé par toutes les organisations syndicales sauf la CGT. En 2007, un avenant, étendant les principes généraux de l'accord précédent aux ouvriers professionnels, a été signé par toutes les organisations syndicales sauf la CGT.

La description du système en vigueur jusqu'alors met en lumière une confrontation intergénérationnelle à travers la prise en compte du diplôme et de l'ancienneté dans l'établissement des classifications professionnelles. Depuis une quinzaine d'années, au sein du groupe

5/ Avant le déploiement des nouveaux accords qui introduisent des niveaux supplémentaires, l'échelle des coefficients pour les ouvriers se déroule ainsi : 170, 180, 190, 200, 215, 225, 240, 255, 270, 285. 
des ouvriers qualifiés, un nombre grandissant de jeunes titulaires de baccalauréats professionnels est en effet amené à côtoyer quotidiennement des générations plus anciennes, où dominent largement les titulaires de CAP. Cette situation permet de s'interroger sur les conséquences de l'arrivée de ces jeunes bacheliers professionnels, sur les changements occasionnés dans les rapports entre générations ouvrières et le bouleversement des hiérarchies ouvrières qui en découle. L'« ordre de l'ancienneté », qui renvoie au fait que les acteurs désignent ce critère de l'ancienneté comme décisif pour légitimer l'établissement des hiérarchies dans la qualification ouvrière ${ }^{6}$, paraît en effet mis à mal par la manière dont les bacheliers professionnels ont été intégrés dans les systèmes de gestion des classifications en vigueur. Cette culture d'atelier qui valorise l'expérience comme source des connaissances qui viennent légitimer la qualification se transmet pourtant au sein de la population ouvrière. Nous verrons ensuite comment l'introduction d'une classification par la compétence renouvelle ces oppositions entre générations en instaurant une situation de déni de l'expérience. Finalement, nous nous interrogerons sur l'effectivité du changement proposé en identifiant des formes de résistance à la remise en cause de l'ordre de l'ancienneté dans l'atelier.

\section{LA GRILLE DE 1975 : LE CHOC DES COEFFICIENTS OU L'OPPOSITION OBJECTIVE DES GÉNÉRATIONS}

Dans le système mis en place en 1975 (cf. encadré 2), le diplôme est un critère classant à l'embauche, du moins tant que celui-ci a été acquis avant l'affectation dans l'entreprise, qu'il relève du même secteur industriel et, dans une certaine mesure, que le poste occupé correspond à la formation du salarié. Ce critère reste déterminant dans la suite du parcours professionnel, puisque les coefficients sont ordonnés sur une "échelle unique et continue de coefficients » (extrait du texte de l'accord de 1975) et que tout retour en arrière est dans les faits pratiquement exclu. Sur cette échelle qui va théoriquement de 140 à $285^{7}$, le titulaire d'un CAP ou d'un BEP industriel ne doit pas être accueilli en dessous du coefficient 170 . Le titulaire d'un baccalauréat professionnel industriel ne doit pas, lui, être accueilli en-dessous du coefficient 215. En outre, si le diplôme

6/ Ceci ne signifie donc pas qu'il y aurait effectivement une prééminence systématique de ce critère dans l'évaluation et la promotion, conformément au modèle de gestion qu'Eyraud et al. (1989) avaient qualifié de « gestion interne des classifications».

7/ En réalité, dans l'entreprise étudiée, le seuil d'accueil minimal (celui d'un apprenti) est fixé au coefficient 160 ; exceptionnellement le coefficient 305, en principe réservé à la filière techniciens/agents de maîtrise, est utilisé pour classer des ouvriers en toute fin de carrière. 
est un critère relativement récent dans la détermination de la qualification ouvrière (Prost, 1981 ; Charlot et Figeat, 1985), la définition de l'ouvrier qualifié, qu'elle soit donnée par des représentants de la direction ou par les ouvriers eux-mêmes, repose actuellement fortement sur la possession d'un diplôme : selon l'opinion largement partagée dans l'atelier, « un professionnel, c'est quelqu'un qui a un diplôme».

$\mathrm{Au}$ sein de l'atelier, un deuxième mode de légitimation de la qualification au travail, l'expérience, se juxtapose au diplôme. En fait, cette dimension est plutôt référée au travers du terme « ancienneté », considéré comme un quasi-synonyme. C'est en effet par l'ancienneté que l'on peut obtenir l'accumulation de situations de travail variées, qui favorise l'apprentissage progressif des "trucs», des « tours de main », des « ficelles du métier » ou autres « recettes » qui permettent à l'ouvrier expérimenté de faire face à des situations de travail inattendues (Brucy, 2007). Du point de vue des ouvriers, l'ancienneté est donc la seule véritable manière d'objectiver l'expérience acquise dans le travail.

\section{- Le poids de l'ancienneté et du diplôme en question}

Pour les ouvriers qualifiés âgés, ces deux modes de légitimation de la qualification, le diplôme et l'expérience, venaient se renforcer mutuellement. Or, à partir du début des années 1990, cette synergie est progressivement remise en cause avec l'embauche de jeunes titulaires de baccalauréats professionnels sur des postes d'ouvriers qualifiés.

Un regard sur la répartition des coefficients en fonction de l'âge, sur deux des sites d'enquête ${ }^{8}$, permet de cerner l'ampleur du problème. Sur le site B (figure 1), le coefficient 215 rassemble à lui seul un tiers de l'effectif des ouvriers qualifiés $(33,6 \%$ des OP du site à la date de l'enquête). On observe une distribution nettement bimodale avec, d'une part, de nombreux jeunes âgés de moins de 30 ans et titulaires d'un bac professionnel, et, d'autre part, des anciens âgés de plus de 40 ans et titulaires d'un CAP. Pour les premiers, le coefficient 215 correspond au coefficient d'embauche tandis que, pour les seconds, embauchés initialement au coefficient 170 (parfois à 180), il représente un palier supplémentaire de leur évolution professionnelle (après qu'ils aient atteint les coefficients 180, 190 et 200).

8/ Ces données nous ont été communiquées par les responsables des ressources humaines des sites B et C. Il s'est avéré impossible d'obtenir les mêmes données sur les sites A et D. 


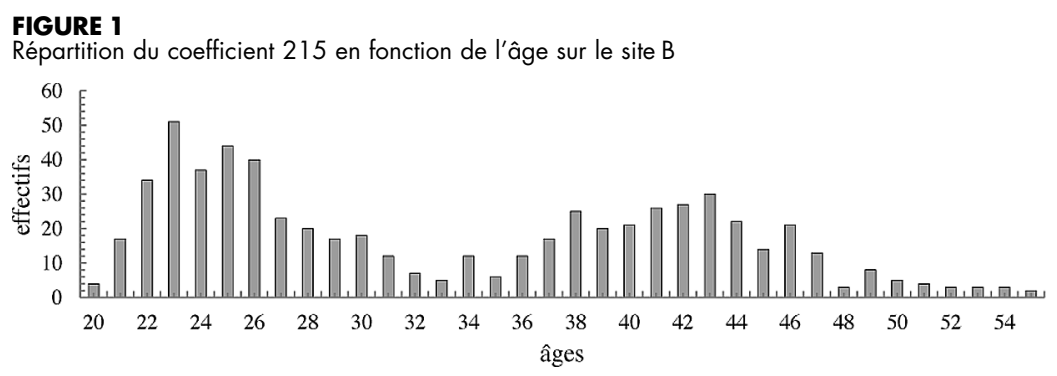

Sur le site C, les titulaires du coefficient 215 sont proportionnellement moins nombreux $(17,9 \%)$ alors que les titulaires des coefficients 225 et 240 représentent $44,2 \%$ des OP du site à la date de l'enquête; dès lors, la distribution sur le coefficient 215, bien que vraisemblablement bimodale, est moins parlante. Le phénomène se décèle plus clairement si l'on regarde les coefficients 225-240 (figure 2).

\section{FIGURE 2}

Répartition des coefficients 225-240 en fonction de l'âge sur le site C

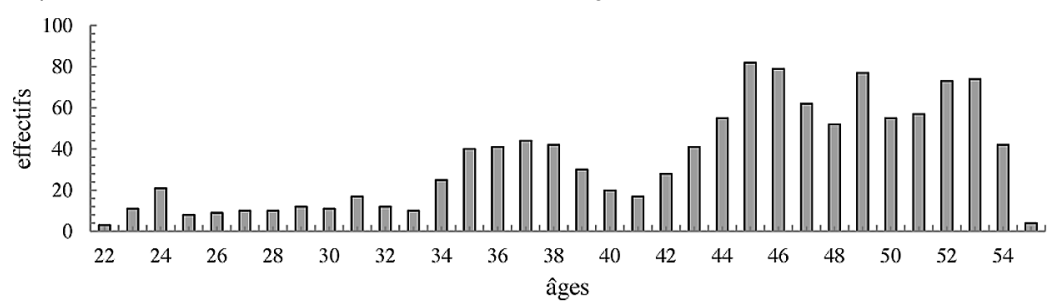

Ici, le premier pic se situe autour de 36-37 ans avec une queue de distribution très étalée vers les âges les plus jeunes, ce qui correspond en partie à de jeunes bacheliers ayant acquis un ou deux coefficients depuis l'embauche, tandis le second pic se situe autour de 50 ans, correspondant aux anciens entrés avec un CAP. En fait, il s'agit bien là d'une structure semblable à la précédente malgré un décalage dû aux différentes vagues de recrutement. En effet, la population du site $C$ est âgée de 5 à 6 ans de plus que celle du site $B{ }^{9}$, ce qui explique en grande partie la disparité observée dans la répartition des coefficients.

Au sein des mêmes coefficients - 215, 225, 240 - deux populations d'âge, d'ancienneté et de niveaux de diplôme nettement

9/ Les données fournies par les directions des sites indiquent, pour la population des ouvriers qualifiés au moment de la réalisation de l'enquête, 38,5 ans pour l'âge moyen et 40 ans pour l'âge médian sur le site B contre respectivement 43,5 ans et 46 ans sur le site $C$. 
différents se côtoient donc. Cette distribution bimodale des coefficients traduit la coexistence problématique de deux périodes du système de relations entre formations et emplois. Il semble qu'il y ait eu, lors de la création du baccalauréat professionnel, et surtout lors de son intégration dans la structure de classification préexistante au sein de la métallurgie, une surévaluation du niveau des postes pour lesquels les bacheliers professionnels étaient susceptibles d'être embauchés. Au départ pensés comme des «techniciens d'atelier » destinés à sortir rapidement de l'atelier pour accéder à de véritables postes de techniciens (Campinos-Dubernet, 1995 ; Veneau et Mouy, 1995), les jeunes «bacs pros» sont en fait devenus des ouvriers qualifiés venant se substituer aux titulaires de CAP-BEP à travers un processus de dévaluation des diplômes (Eckert, 2005).

Ces bacheliers professionnels ont ainsi été embauchés directement au niveau 215, qui représentait un niveau élevé dans la hiérarchie professionnelle des postes ouvriers : le niveau P3 ${ }^{10}$. Mais loin de quitter rapidement l'atelier, ils se sont en fait stabilisés dans des emplois d'ouvriers qualifiés. Cette surévaluation des emplois auxquels ils pouvaient potentiellement accéder crée donc un profond décalage entre la structure des diplômes obtenus, temporellement située, et la structure des coefficients dans l'entreprise au moment de l'enquête. Alors qu'ils occupent des postes d'ouvriers qualifiés « de base », ils se voient d'emblée dotés d'un coefficient élevé auquel les anciens ne parvenaient que bien plus tard.

\section{Le dénigrement des bacheliers professionnels par les anciens}

Or, pour les ouvriers, le coefficient, souvent assimilé à un 《 grade » ou un « galon» (« il est $215 »$ ou « je suis $255 »)$, est censé être une reconnaissance de la valeur au travail (Cousin, 2007, p. 198). Il a ainsi une efficacité bien réelle dans les rapports quotidiens entre ouvriers et une importance centrale dans l'établissement des hiérarchies professionnelles au sein de l'atelier.

10/ L'enjeu symbolique autour de ce coefficient 215 est extrêmement fort. Autrefois niveau élevé dans la hiérarchie des ouvriers qualifiés, il sert au moment de l'enquête de frontière entre les deux catégories ouvrières dans la mesure où toute une gamme de coefficients (globalement du 180 au 200) est devenue commune à la catégorie des ouvriers professionnels et à celle des opérateurs. Dès lors, pour ceux qui démarrent comme opérateur, ce coefficient est le niveau à atteindre pour espérer obtenir une classification de « professionnel », et obtenir « une protection contre le risque d'un retour en chaîne » (Hatzfeld, 2004, p. 28). Les réformes managériales engagées dans cette entreprise tendent justement à remettre en cause ce principe de protection conférée par le niveau 215, en particulier via la prolongation de la zone de recouvrement entre les deux catégories ouvrières et l'élargissement des possibilités de déclassement de la catégorie « ouvriers professionnels » vers celle des « opérateurs » (Misset, 2009). 
Avec l'arrivée des bacheliers professionnels, la balance s'est brutalement mise à pencher du côté du diplôme au détriment de l'expérience.
Dès lors, les anciens refusent l'équation qui voudrait qu'un CAP ou un BEP obtenu il y a 20 ans et associé à une expérience professionnelle longue soit l'équivalent formel d'un bac professionnel récent et quasiment dépourvu d'expérience professionnelle. À leurs yeux ce dernier ne peut prévaloir pour venir légitimer une position d'ouvrier qualifié dans l'atelier. On pourrait donc dire qu'avec l'arrivée des bacheliers professionnels, la balance, jusque-là équilibrée entre les deux modes de légitimation de la qualification au travail, s'est brutalement mise à pencher du côté du diplôme au détriment de l'expérience. Un interviewé critique ainsi avec force le fait que deux années d'études supplémentaires («Si on peut appeler ça des études ! » ironise-t-il) permettent aux bacheliers professionnels d'arriver au même niveau que lui après une décennie de travail :

\begin{abstract}
« Moi j'ai CAP-BEP, donc moi je suis rentré 180 donc P1, donc maintenant, je suis 255 et quelque part pour arriver au niveau 215, qui est le niveau des embauchés des bacs pros, il m'a fallu 11 ans quand même. Alors que eux ils arrivent... [...] Mon évolution... j’ai été la chercher, c'est vrai aussi, j'ai été la chercher. Alors que maintenant... À l'époque où j'ai commencé, un coef 215 , pour moi c'était quelqu'un qui avait une grande maîtrise de l'atelier, c'était quelqu'un... c'était un caìd. Alors que maintenant on me dit un 215, pour moi, ça veut rien dire. Pour moi, ça veut rien dire. Pour moi maintenant, le coefficient n'est plus le reflet de la valeur de la personne. » (Francis, 38 ans, CAP-BEP, conducteur d'installation).
\end{abstract}

Alors que les anciens cherchent à fonder leur propre légitimité d'ouvriers qualifiés sur leur parcours scolaire en CAP, qu'ils se vivent comme une véritable aristocratie ouvrière, ils contestent le fait que le diplôme des jeunes soit un critère suffisant pour légitimer leur position, en mettant en cause les savoirs dispensés à l'école et la qualité de la formation suivie. Ils établissent ainsi une distinction nette entre les titulaires de CAP représentant « l'élite des réprouvés » (Grignon, 1971) d'une époque révolue, et les jeunes bacheliers professionnels, orientés par défaut en filière professionnelle, qu'ils refusent de considérer comme leurs dignes successeurs. En outre, à travers leurs discours, c'est aussi une critique plus générale d'une forme de « désouvriérisation » du lycée professionnel (Beaud, 1996) que l'on perçoit.

\footnotetext{
« Ils savent pas lire un plan. On leur ouvre, la pièce elle est là, il faut ressortir la pièce là, il faut la dessiner, il faut la relancer à la maintenance centrale pour qu'on la change, ils savent pas le faire... "Ouais mais moi j'ai appris sur le programme là X Y" "oui mais on travaille avec autre chose" "ah ouais, je sais pas faire"... c'est un truc, c'est impensable pour une personne qui a eu son CAP, qui a appris pendant trois ans, parce que moi mon CAP, c'était en trois ans à l'époque, j'avais $8 \mathrm{~h}$ de dessins par semaine, c'est la base de notre métier, notre métier de mécanicien [...]. » (Francis, 38 ans, CAP-BEP, conducteur d'installation).
} 
« Aujourd'hui c'est des bacs professionnels, mais même un bac MSMA ${ }^{11}$, il sait pas travailler. Il sait pas, il y a beaucoup de choses qu'il sait pas. Soit qu'il a pas appris, soit qu'on lui a pas appris, mais ils savent pas. Ils savent pas travailler. La plus simple des choses, ils savent pas le faire. [...] Ils savent pas comment on perce, ils savent pas comment on lime... ils savent pas comment... ça ils savent pas faire. Ils savent... se servir d'une lime, ils savent pas faire. C'est vraiment la base qu'ils ne savent pas faire. [...] Sincèrement je sais pas ce qu'ils peuvent apprendre à l'école. En fait, faut tout leur apprendre derrière. » (Gilles, la cinquantaine, CAP, chef d'atelier, ancien ouvrier qualifié).

À travers une référence, directe ou détournée, au « compagnonnage », les anciens se montrent également nostalgiques d'une époque où « on apprenait à travailler avec des coups de pieds dans le derrière », d'un temps où « ça marchait à la baguette », de la soumission d'un jeune « apprenti » à l'autorité d'un «maître » possédant un savoir-faire qu'il lui communique progressivement, d'une place dans l'atelier qui n'était pas contestée.

\footnotetext{
« Quand je suis arrivé, tout jeune, "commence déjà à apprendre à ranger la caisse"... C'est comme ça que j'ai appris à travailler. Et "oui monsieur"... il y a moins maintenant le respect. "Tu bosses un peu, je vais boire mon café", il allait boire son café, il revenait "t'as passé une meule là-dessus ?", il regardait le travail. Ils font plus ça. » (Pascal, la cinquantaine, CAP, maintenancier).

« Moi quand je suis arrivé, on m'a mis avec un ancien, un vieux, on m'a dit... je me suis mis à... parce que moi j'étais motivé, donc je me suis mis avec le vieux et le vieux il m'a dit "tu prends ma caisse à outils". Je l'ai pris, je lui ai portée. Il me faisait voir, mais c'est moi qui... Bon je faisais un peu le sale boulot mais... ça ne faisait rien. Aujourd'hui, les jeunes ils acceptent pas trop ça. Et bon les vieux ils le font plus trop non plus.» (Gilles, la cinquantaine, CAP, chef d'atelier, ancien ouvrier qualifié).
}

La virulence des propos tenus par les anciens sur les bacheliers professionnels se comprend mieux lorsque l'on voit à quel point leur position dans l'atelier est remise en cause par les systèmes de gestion en vigueur. Ils ont le sentiment que la situation précédemment décrite en matière de répartition des coefficients annule la valeur de leur expérience tout en diminuant, par anachronisme, celle de leur diplôme.

\section{L'INTROUVABLE CONFLIT DE GÉNÉRATIONS ${ }^{12}$}

Toutefois, cette situation d'opposition objective entre les deux générations n'engendre pas véritablement un « conflit de génération», au sens fort du terme. Il faut en effet chercher les sources

11/ Maintenance des systèmes mécaniques automatisés, spécialité de baccalauréat professionnel.

12/ Nous empruntons l'expression à N. Flamant (2004) 
La valorisation de l'ordre del'ancienneté reste largement partagée dans I'atelier et transmise des aînés aux plus jeunes. d'opposition entre les générations dans les caractéristiques des modes de gestion de la population et les réorganisations professionnelles menées, davantage que dans les caractéristiques propres des générations (Flamant, 2005 ; Huyez-Levrat, 2007). Malgré le bouleversement des hiérarchies professionnelles que provoque l'arrivée des bacheliers professionnels au niveau 215, la valorisation de l'ordre de l'ancienneté reste largement partagée dans l'atelier et transmise des aînés aux plus jeunes.

\section{La transmission de l'ordre de l'ancienneté}

Ainsi, les jeunes OP reconnaissent généralement la supériorité professionnelle des anciens et admettent sans difficulté le critère de l'ancienneté comme un critère essentiel de légitimité et de hiérarchisation dans l'univers de l'atelier, qui permet de décider de l'ordre des promotions ou de l'attribution des tâches ingrates aux jeunes générations, puisque les connaissances obtenues par le diplôme doivent nécessairement être complétées par un apprentissage «sur le tas».

«Pour faire le tour, il faut déjà un sacré nombre d'années. [...] Une personne qui a 25 ans d'ancienneté dans l'outillage, elle va être beaucoup plus connaisseuse que quelqu'un qui a 10 ans d'expérience. » (Frédéric, 26 ans, baccalauréat professionnel, ajusteur-outilleur).

« Ils sont moins diplômés mais je veux dire vis-à-vis de l'expérience professionnelle, c'est quand même 20 ans d'ancienneté, ils ont quand même vécu et vu beaucoup plus de choses que moi. " (Marc, 25 ans, baccalauréat professionnel, conducteur d'installation).

Ils remarquent donc le malaise suscité par la distribution des coefficients selon l'âge et partagent le jugement des anciens sur celle-ci, comme en témoignent ces deux extraits d'entretien :

« Je connais un ancien. Il est rentré avec un BEP, il a 20 ans d'ancienneté, il est 215 ... Vous, vous arrivez, vous avez 0 année d'ancienneté, vous êtes 215 ... [...] Je me mets à leur place, c'est normal. C'est normal que le mec... et en plus le gars, ça fait 20 ans qu'il est là, il est 215 , et il doit former celui qui arrive et qui est 215 aussi. C'est normal que ça leur plaise pas. [...] Je suis rentrée 215, j'ai entendu "ouais c'est pas parce que t'as un bac... la preuve tu sais pas dépanner toute seule...". On leur dit "attends, j'ai pas choisi, j'ai pas demandé qu'ils me mettent là", ça s'arrête là. Sur le principe, ils sont un peu dégoûtés, eux ils sont largement meilleurs que nous et ils ont le même coef que nous. » (Valérie, 29 ans, baccalauréat professionnel, électromécanicienne de maintenance).

« Je suis à $215 \ldots$ et je bosse avec des mecs, qui ont 25 ans de boîte et qui sont à 200. Moi petit jeune, j'étais un peu gêné par rapport... le mec il me formait mais j'étais un grade au-dessus sur la fiche de paie, ça choque toujours un peu. Ça choque. » (Fabrice, 26 ans, baccalauréat professionnel, conducteur d'installation). 
En effet, ils ont concrètement besoin des anciens pour faire face à certaines difficultés du travail et savent qu'ils verraient vite le « revers de la médaille » s'ils prenaient de haut les anciens en mettant en avant leur niveau de diplôme ou leur coefficient. Notamment parce que ces jeunes ont été formés par des anciens - même si ce n'est plus sous le principe du compagnonnage - et que ces derniers ont ainsi pu prouver leur supériorité technique soit en pratiquant une rétention d'informations handicapante pour les jeunes:

\begin{abstract}
«Le parrainage, c'est bien oui et non, parce que si la personne veut pas nous montrer, elle fera tout pour pas nous montrer. Donc on pourra jamais évoluer. La plupart des gens sont comme ça, c'est un peu dommage. Par exemple, quand il y a quelqu'un qui est vachement plus performant, lui il fera exprès d'aller vite, de faire des manips très vite pour qu'on comprenne pas, de peur qu'on lui prenne sa place peut-être ou je sais pas. Alors donc, moi je me suis accroché pendant un sacré moment. » (Emmanuel, 31 ans, CAP, électromécanicien de maintenance).
\end{abstract}

Soit au contraire en les partageant:

«Le mec qui m’a formée quand je suis arrivée, il a 20 ans d'ancienneté, et des fois c'est hallucinant: "j'ai une panne là", "tu grattes le tas de copeaux qu'il y en a en bas à gauche de l'unité, tu verras, il y a un micro en dessous, c'est ça"... "qu'est-ce qu'il me raconte", vous y allez, pas convaincue, vous grattez le tas de copeaux, ah oui il y a un micro, le mec il connaît du bout des doigts l'atelier. » (Valérie, 29 ans, baccalauréat professionnel, électromécanicienne de maintenance).

Il ne faut bien sûr pas en déduire que les relations entre jeunes et anciens sont totalement pacifiées. Il arrive ainsi que les jeunes reprochent aux anciens d'être parfois "vieille école», de se montrer " bourrus », " limite désagréables », et surtout excessivement regardants sur les capacités professionnelles manifestées par les jeunes. Mais les tensions qui pourraient en découler semblent régulées si les jeunes montrent ostensiblement leur désir d'apprendre et surtout s'ils valident l'ordre de l'ancienneté en autorisant les anciens à faire des réflexions sur la qualité du travail ou les modalités de sa réalisation.

«Là aussi c'est quelque part une qualité à avoir parce qu'il y a des gens, quand vous arrivez, et que vous avez 20 ans dans une usine et que les anciens ils ont 30 ans d'ancienneté, eux ils sont vraiment pointilleux, ils vont faire recommencer un travail 3 ou 4 fois. Faut savoir accepter aussi. » (Frédéric, 26 ans, baccalauréat professionnel, ajusteur-outilleur).

À l'inverse, ces relations intergénérationnelles, relativement apaisées quand un respect de l'ordre de l'ancienneté est ainsi affiché, se 
dégradent lorsqu'il est remis en cause par l'attitude des jeunes ou par des promotions qui semblent «court-circuiter» le schéma normal de carrière et suscitent la « jalousie».

«Un ancien, faut pas lui dire ce qu'il a à faire, le jeune, il se met plus en... en comment... c'est à lui de se remettre en question mais sûrement pas à l'ancien. Donc après s'il est tombé sur un têtu qui ne se laisse pas faire, forcément ça fait des étincelles. Quelqu'un qui est assez maniable... L'ancien, il va dire "toi tu fais ça" parce qu'il est un petit peu... on va dire il est un petit peu le chef, il va dire "tu fais ça", si le jeune en face, il est têtu, il dit "ouais, faut pas faire comme ça", ça y est, tout de suite, c'est la guerre quoi. Dans une équipe, il en faut pas plus à mon avis pour... déclencher une catastrophe. » (Franck, 26 ans, baccalauréat professionnel,

Le non-respect de l'ordre de l'ancienneté semble être le principal facteur générant des

tensions ouvertes entre les générations d'ouvriers qualifiés. metteur au point).

Ainsi le non-respect de cet ordre de l'ancienneté semble être le principal facteur générant des tensions ouvertes entre les générations d'ouvriers qualifiés.

\section{La difficile reconnaissance des jeunes promus}

En outre, si la situation décrite précédemment ne peut être lue sous l'angle du conflit de génération, c'est aussi parce que cette question des coefficients possède un deuxième versant problématique, celui de la reconnaissance de la qualité du travail effectué par les jeunes opérateurs promus ouvriers professionnels en interne. En effet, ceux-ci, initialement embauchés aux coefficients inférieurs de la grille, ne parviennent pas à rattraper les coefficients détenus par les jeunes bacheliers professionnels quand bien même ils occupent des fonctions similaires dans l'entreprise. Ainsi, Nicolas, jeune moniteur ${ }^{13}$ titulaire du coefficient 190, initialement embauché comme opérateur ${ }^{14}$, se plaint de l'absence de justice que constitue la répartition des coefficients:

« Ils ont embauché des moniteurs tout jeunes qui sortaient du bac pro... Moi je suis arrivé comme moniteur, j'ai pas eu le coefficient 190 tout de suite, je suis rentré moniteur 180 et je voyais des gens qui sortaient du bac à 20 ans, ils étaient déjà 215. 215, ça calme. Il a déjà 150 euros de plus que vous nets. Et faut avoir le sourire. Parce qu'il fait exactement le même travail que vous. » (Nicolas, 29 ans, niveau DEUG, moniteur).

\footnotetext{
13/ Les moniteurs sont des ouvriers qualifiés chargés d'une mission de contrôle de qualité et des retouches sur les chaînes de production ; leur domaine de compétences s'élargit dans les faits à une gestion informelle des opérateurs qui y travaillent (formation, remplacement, surveillance...).

14/ Malgré son niveau de diplôme (après un baccalauréat $S$, il a échoué en deuxième année de DEUG de physique), Nicolas a été embauché au plus bas niveau des grilles de classification de la métallurgie: en effet, selon les accords en vigueur, comme il ne dispose pas d'un diplôme professionnel du secteur industriel, il n'a officiellement aucun diplôme reconnu pour une embauche sur un poste d'ouvrier. Tout se passe comme s'il n'avait « même pas un CAP ».
} 
Mais la critique de Nicolas ne s'arrête pas aux jeunes bacheliers professionnels. La reconnaissance tacite de l'ordre de l'ancienneté ne semble en effet être mise à mal que par ceux qui, comme lui, sont à la fois démunis de la ressource de l'ancienneté (jeunes) et de celle du diplôme (promus en interne). Estimant que l'ancienneté ne justifie en rien un coefficient plus élevé ( $«$ Le bagage technique, comme je vous ai dit, si on a envie d'apprendre, on apprend très vite. »), Nicolas affirme d'ailleurs que cette question des coefficients est dans son équipe « une grosse source de conflit»:

« J'ai un exemple, un ancien qui a 35 ans de boîte, qui se fait plus de 2000 euros nets par mois et puis moi qui me fais même pas 1400 par mois. Et l'ancien fait pas grand-chose et a normalement exactement la même charge de travail que moi sur la journée. Ce qui est pas du tout le cas. Il boit beaucoup de cafés et fait pas grand-chose. » (Nicolas, 29 ans, niveau DEUG, moniteur).

La confrontation des générations se complexifie alors puisque selon les cas, les anciens peuvent devenir les alliés indirects des jeunes promus contre les prérogatives de bacheliers professionnels ou inversement les adversaires potentiels de jeunes non diplômés mais qui s'estiment plus à l'aise qu'eux. Les anciens peuvent en outre mobiliser l'argument du diplôme contre les « gens du cru » promus en interne et l'argument de l'expérience contre les bacheliers professionnels. Il n'est donc pas très étonnant que les organisations syndicales aient eu des difficultés à avoir une position claire sur la reconnaissance des diplômes lors de la négociation de la nouvelle grille de classification : toute position explicite aurait risqué d'alimenter l'opposition entre jeunes (majoritairement bacheliers professionnels) et anciens (majoritairement titulaires d'un CAP), ainsi qu'entre ouvriers professionnels à l'embauche et ceux issus des rangs des opérateurs.

Chez les anciens comme chez les jeunes promus en interne, la situation en matière de répartition des coefficients engendre la crainte d'une prévalence du diplôme qui les marginalise: "Une grande partie des professionnels, qui ont un CAP d'il y a 30 ans, ils ont évolué avec leur matériel, on leur donnera jamais un coefficient. Pourtant, ils le méritent, par rapport au travail qu'ils ont fait. Mais comme ils ont pas le diplôme, ils auront pas le coefficient. Et c'est ça, la peur de l'accord professionnel » déplore ainsi Pascal. Car, en filigrane, le nouvel accord de classification des ouvriers professionnels négocié dans l'entreprise leur semble entériner une situation de déni de la valeur de l'expérience. À travers celui-ci, la confrontation des générations prend en effet une nouvelle tournure puisque cet accord alimente le clivage entre jeunes et anciens en favorisant la non-reconnaissance des compétences acquises par l'expérience.

Le nouvel accord alimente le clivage entre jeunes et anciens en favorisant la non-reconnaissance des compétences acquises par l'expérience. 


\section{UNE LOGIQUE COMPÉTENCE QUI REJOUE L'OPPOSITIONDES GÉNÉRATIONS?}

Au siège de l'entreprise, l'argumentation de la nécessité de nouvelles grilles de classification repose notamment sur les perceptions ouvrières de l'évolution professionnelle, telles qu'elles ressortent des propos rapportés par certaines études sociologiques réalisées dans l'entreprise ${ }^{15}$, mais aussi telles qu'elles sont identifiées par la hiérarchie. Dans l'ancien système, l'évolution professionnelle serait injuste, à la « tête du client », fonction de la « cote d'amour» ou de la «note de gueule », soumise au " piston », au « copinage du coin », etc. Les dirigeants de l'entreprise défendent donc l'intérêt d'un système « transparent » et « objectif » qui s'opposerait à l'arbitraire et l'opacité du système jusqu'alors en vigueur (cf. encadré 2).

Mais le discours des managers met également en scène une opposition entre jeunes et anciens, justifiant ces nouvelles grilles comme une nécessité face aux attentes des premiers et au fort renouvellement des effectifs :

\footnotetext{
« Les classifications actuelles ne répondent pas aux attentes des salariés, et, en particulier, des 21000 jeunes ouvriers que l'entreprise a recruté depuis 5 ans, qui souhaitent avoir une vision claire, et des possibilités d'évolution et de promotion sociale. » (Extrait d'un document de communication interne).
}

En outre, les anciens sont indirectement visés par une autre série de critiques portant sur des "glissements à l'ancienneté », c'està-dire une progression inexorable déconnectée des performances réelles et de l'investissement au travail des individus, qui serait permise par l'ancien système. Cette critique s'appuie notamment sur le constat qu'un des premiers critères utilisés par les chefs d'équipes et les chefs d'atelier pour décider de l'attribution d'un coefficient est le temps écoulé depuis l'obtention du coefficient précédent. Par ailleurs, certains de ces « glissements à l'ancienneté » ont été contractualisés suite à des grèves: dans un avenant aux accords de classification de 1975, une clause stipule qu'après 8 ans d'ancienneté au coefficient 170 , le salarié obtient automatiquement le passage au coefficient 180, et après 12 ans d'ancienneté au coefficient 180, le passage au coefficient 190 .

15/ En particulier les études sociologiques réalisées à la demande de l'entreprise et portant sur l'intégration des jeunes embauchés sur les sites de production (Moncel et Sulzer, 2006; Misset, Eme et Hinault, 2004) Ces études ont été explicitement citées lors de la convention d'entreprise visant à promouvoir le nouvel accord. 


\section{- Une logique compétence qui constitue un déni de l'expérience}

La nouvelle grille de classification se présente sous forme de référentiels de compétences propres à chaque métier. Ceux-ci sont constitués de tableaux croisant en colonne les différents domaines (maîtrise technique, démarches de progrès, transfert de compétences...) avec les coefficients figurant en ligne. Chaque case, située au croisement d'une ligne "coefficient» et d'une colonne «domaine» décrit des activités (par exemple «isoler les non-conformes », «être polyvalent sur deux postes »...). Pour pouvoir passer au coefficient supérieur, les ouvriers doivent remplir toutes les conditions qui figurent dans les cases d'une même ligne pendant une période minimale de mise en situation de deux à trois ans selon les coefficients.

Dans ce nouvel accord, les signataires définissent la «compétence » comme une «capacité à agir, à réaliser une activité » qui "s'observe dans l'action et se mesure en situation professionnelle». L'évaluation des compétences se fait donc sans revenir sur l'histoire professionnelle de lindividu puisque seules comptent les « compétences » réellement exercées pendant l'année écoulée. Autrement dit, dans la logique mise en oeuvre dans cette grille, toute capacité à réaliser une activité qui n'est pas ou plus mise en cuvre sur la période d'observation n'est pas une compétence. Lors d'une mobilité professionnelle, le salarié perd ainsi les compétences qui étaient maîtrisées dans son ancienne fonction ou son ancien secteur pour se voir repositionner à un niveau potentiellement inférieur. En théorie, l'accord ne permet pas la perte du coefficient (le salaire étant donc maintenu lors d'une mobilité), mais dans ce cas, le coefficient réel devient supérieur au "coefficient théorique » (celui qui est obtenu par rapport aux compétences répertoriées dans le référentiel).

\footnotetext{
«Je vais donner un exemple, un mec qui travaille dans les culasses, je vais le mettre au carter, il a fait du transfert mais il y a des choses qui diffèrent. Donc il a un coefficient, je vais donner un exemple, 240. Il va arriver dans l'atelier là, si on prend la grille comme elle est, qu'on dit qu'on regarde la personne à l'instant $\mathrm{T}$, ce qu'elle fait, ce qu'elle est capable de faire. Le gars arrive, ce transfert-là, il le connaît pas bien, on va lui dire "ben ouais mais écoute, là aujourd'hui tu es évalué comme un 190". Et ça on a beau expliquer aux gens, leur dire, "tu vas vite rattraper la marge, ton coefficient il ne bouge pas, tu restes 240", imaginez-vous psychologiquement l'impact que ça fait. » (Didier, gestionnaire du personnel).
} 
C'est alors au salarié de combler l'écart avant de pouvoir espérer gagner à nouveau des coefficients ${ }^{16}$. Les notions de «transférabilité », ou de «portage des compétences», initialement contenues dans le modèle de la «logique compétence » (Monchatre, 2004; Tallard, 2001) apparaissent pour le moins vidées de leur substance au regard de l'application concrète de ce modèle. Cette définition de la « compétence » contient bien un déni de l'expérience, puisque le salarié doit régulièrement prouver sa qualification (Brucy, 2007 ; Monchatre, 2002 ; Richebé, 2002). L'ordre de l'ancienneté, autrefois «principe de justice » (Brucy, 2007, p. 294), déjà sérieusement ébranlé par l'arrivée de bacheliers professionnels, serait-il cette fois anéanti par la conception de la compétence professionnelle que véhicule cet accord?

\section{Des anciens bloqués, des jeunes avantagés?}

Cette conception de la « compétence » promue par les dirigeants au siège de l'entreprise - une évaluation de la compétence effectuée «à l'instant $\mathrm{T}$ » complètement « faussée » selon beaucoup d'interviewés - et les implications qui en découlent en matière de reconnaissance de la qualification acquise à travers l'expérience suscitent sur le terrain de nombreux remous, tant chez les managers locaux que chez les délégués syndicaux.

Les managers de proximité rencontrés ont le sentiment que la « compétence », telle qu'elle est formulée dans l'accord, nie totalement l'apport spécifique des anciens dans la réalisation des activités de travail, apport réel même s'il est difficilement formalisable ${ }^{17}$. Ils estiment alors que les dirigeants de l'entreprise font le pari de ne miser que sur les jeunes (leur « adaptabilité beaucoup plus grande », leur «bagage plus élevé ») pour construire l'avenir de l'entreprise, pari qui leur semble à la fois injuste et risqué. En effet, leurs propos trahissent le fait qu'ils partagent en partie l'ordre de l'ancienneté qui doit, selon eux, prévaloir pour l'attribution des postes et les promotions notamment; l'argument d'une « culture ouvrière » qui valorise l'expérience et qu'il faudrait respecter sous peine de mettre en péril

16/ En remplissant les conditions indiquées dans le référentiel de compétences pour chaque coefficient manquant entre son coefficient théorique et son coefficient réel, et ce pendant les périodes minimales de mise en situation entre deux coefficients, puisque le saut de coefficient n'était possible qu'au moment du déploiement de l'accord. L'évaluation des compétences mises en œuvre est établie annuellement lors d'un entretien avec le chef d'équipe. Il existe une possibilité de recours pour le salarié insatisfait de son évaluation. Il est également à noter que le contenu des référentiels de compétences ne fait pas partie de l'accord de classification proprement dit et peut donc être modifié unilatéralement par l'employeur.

17/ Ils se sont alors emparés de l'enquête comme chambre d'écho à leurs récriminations, espérant que ce travail pourrait servir à réorienter les principes défendus au siège de l'entreprise. 
le fragile équilibre des ateliers est, à leurs yeux, recevable. En outre, ils expriment à de multiples reprises leurs « craintes » qu'un mouvement de contestation se mette en place par rapport à cette question de la prise en compte du «passé » professionnel de l'individu.

\begin{abstract}
« Après oui effectivement, il y a certaines choses en fonction des dossiers, on va regarder un peu l'âge du candidat, on se dit ok, son ancienneté... pour dire "voilà cette personne a 23 ans, l'autre il en a 40", celle qui en a 40 , c'est peut-être la dernière chance qu'il aura peut-être, donc on est peut-être un peu plus... Celui qui a 23 ans, qui a un an d'ancienneté, on a repéré qu'il était bon, ce candidat, on peut peut-être lui expliquer qu'il faut peut-être un petit peu patienter pour avoir une certaine reconnaissance. Voilà. » (François, gestionnaire du personnel).

« Les OP sont aussi très attachés sur la forme à la reconnaissance de leur expérience professionnelle, ne pas en tenir compte présente un risque social important. » (Extrait d'un argumentaire envoyé par les managers du site B aux dirigeants du siège pendant la phase de négociation).
\end{abstract}

Les délégués syndicaux s'empressent d'ailleurs d'alimenter ces craintes d'une «levée de boucliers », à l'instar de Pascal, délégué du personnel CFDT, qui avertit :

\begin{abstract}
«Je leur ai dit "votre futur peut-être légendaire accord pour les professionnels, il est pas signé encore. Vous savez, d'autres usines vont nous suivre, si la CFDT signe pas en centrale... faudrait pas vous demander, qu'est-ce qui se passe? Qu'est-ce qu'on a fait de mal ?" Ils s'interrogent quand même, ils pensent pas qu'à rigoler. » (Pascal, la cinquantaine, CAP, maintenancier).
\end{abstract}

Dans l'optique des managers locaux comme dans l'esprit des militants syndicaux, si l'accord est appliqué tel quel, la menace d'un mouvement social chez les ouvriers professionnels est donc réelle. Cette prédiction ne se réalise pourtant pas. Sans surprise, la CGT refuse de signer le nouvel accord de classification, mais celui-ci est finalement signé par cinq organisations syndicales sur six présentes dans l'entreprise, la CFDT prenant quinze jours de réflexion avant d'apposer sa signature. Lors du déploiement, si quelques mouvements épars (débrayages, pétitions...) ont lieu, aucune contestation massive ne prend place. En effet, face à ces questions, les intérêts apparaissent nettement clivés, en fonction de l'âge et du parcours professionnel.

Bien évidemment, le déni de la valeur de l'expérience par l'entreprise fragilise davantage les anciens, qui déplorent la disparition des modalités anciennes d'établissement des hiérarchies ouvrières, les essais P1, P2, et P3. Chez beaucoup d'ouvriers professionnels interviewés prévaut une forme de fatalisme («ce qui tombe, ça sera 
accepté, c'est tout », "s'ils ont décidé, ils feront ce qu'ils voudront »), qui ne les empêche pas de critiquer le principe d'une évaluation par la compétence réalisée dans l'ici et maintenant du poste occupé ; comme le réclame Pascal, " pour les pros, la grille doit se calculer sur la carrière. » Les anciens ont logiquement beaucoup plus à perdre à cette absence de prise en compte du passé professionnel de l'individu, et regrettent que des jeunes puissent les surpasser sur la période prise en compte.

\begin{abstract}
«Ça peut pousser vachement vite quelqu'un alors que l'autre a plus montré au cours des années son savoir-faire. Quelqu'un qui va bien tomber dans la grille, qui a eu un coup de chance de faire une suggestion d'amélioration là-dessus, qui a eu un coup de chance de participer à un cercle de qualité, qui a eu un coup de chance qu'on l'a pris pour faire du $5 \mathrm{~S}^{18}$ le samedi, lui il va tout remplir sa grille bien. Alors qu'une autre personne qui s'est investie pendant des années, qui a fait des tas de trucs positifs, mais qui a pas participé à un groupe de progrès, du coup n'a pas eu de coef. Elle a pas eu de coef et l'autre en a eu deux d'un coup, et il vient d'arriver. Moi ça m'a un peu choqué.» (Serge, 43 ans, CAP, moniteur).
\end{abstract}

D'autres interviewés, en particulier les jeunes promus en interne qui affichaient un discours critique sur les anciens, trouvent au contraire un intérêt incontestable aux nouvelles grilles et attendent avec impatience qu'elles s'appliquent à eux. C'est par exemple le cas de David qui n'hésite pas dans l'entretien à reprendre les critiques managériales sur les « glissements à l'ancienneté » sans rapport avec les performances réelles des OP :

« Un coefficient, il est pas par rapport à l'expérience. On va dire, pour moi. L'expérience propre... par rapport à ce que peut faire la personne ou la qualité qu'elle fournit, c'est par rapport à son âge, c'est tout. » (David, 27 ans, CAP, retoucheur).

Pour les mêmes raisons, Nicolas souhaiterait explicitement qu'il soit possible de remettre en cause le coefficient d'un individu ${ }^{19}$ :

\footnotetext{
«Le seul problème c'est que dans une entreprise comme ça, le coefficient, quand vous l'avez, vous le perdez plus! Il y en a certains qui se poseraient beaucoup de questions. D'ailleurs je trouve ça bête que justement le retour en arrière ne soit pas possible. Parce que ça motiverait beaucoup les gens à travailler. » (Nicolas, 29 ans, niveau DEUG, moniteur).
}

18/ Démarche participative concernant les espaces de travail.

19/ Ce que ne permet pour l'instant ni l'accord de branche de 1975, ni le nouvel accord d'entreprise, bien que cela soit en adéquation avec la logique qui y est promue. 
Les jeunes, promus ou bacheliers, semblent donc favorisés par le système mis en place et les managers locaux admettent qu'ils sont nombreux à avoir gagné deux ou trois coefficients lors de sa mise en place quand les ouvriers professionnels âgés de plus de 40 ans ont été les grands « laissés pour compte» de la logique compétence.

«Les anciens se sentent rabaissés, et les plus jeunes vont vouloir bouffer on va dire les anciens pour pouvoir grimper, c'est normal, et on a plus de mal avec les anciens. » (Michel, 48 ans, CAP, chef d'équipe, ancien ouvrier professionnel).

«Au niveau des anciens, ça a pas mal râlé parce que ils ont pas le sentiment que tout ce qu'ils ont fait jusqu'à maintenant est pris en compte, on redémarre à plat quoi. Et tout ce qu'ils ont fait par le passé, on en tient plus compte. Que ce soit opérateur ou professionnel, ça râle pas mal de ce côté-là.» (Hervé, 38 ans, CAP, chef d'équipe, ancien ouvrier professionnel).

Par « jeunes », il faut toutefois entendre une « classe d'âge » bien plus qu'une "génération», car l'accord, d'une part ne tient aucun compte du retrait progressif de l'investissement des plus âgés (par lassitude, fatigue accumulée, handicaps physiques, manque de formation continue...), d'autre part ne permet pas de capitaliser sur l'expérience acquise au fil d'une carrière qui connaît nécessairement des mobilités. Dès lors, il se pourrait que les jeunes générations déchantent dans quelques années ${ }^{20}$. Un gestionnaire du personnel résume ainsi sa crainte par une formule brutale: "L'avenir, il est plus aux vieux, il est aux jeunes. »

\section{L'appropriation du système en question}

Les données recueillies lors du déploiement des nouvelles grilles de classification permettent toutefois d'envisager un écart entre le système tel qu'il est conçu et son application sur le terrain. Ainsi la force des régulations autonomes (Reynaud, 1988) qui permettent de s'éloigner des principes initiaux ne doit pas être sous-estimée, comme le montre déjà le cas de la sidérurgie (Monchatre, 2002).

À cet égard, il est nécessaire de se pencher sur le rôle crucial des agents de maîtrise, qui ont à mettre en œuvre concrètement la logique compétence décrite plus haut. On trouve souvent chez eux

20/ Même si la conception de la compétence véhiculée dans cet accord devrait conduire logiquement à faire disparaître le critère du diplôme dans l'établissement des hiérarchies professionnelles, celui-ci reste pour l'instant relativement protégé par l'accord de branche et ses « garanties de classement » offertes à ceux qui possèdent un diplôme professionnel industriel et qui occupent un poste en rapport avec leur formation. Notons également que les "glissements à l'ancienneté " contractualisés suite à des grèves ont été maintenus dans les nouveaux accords, à la demande des organisations syndicales. 
un discours qui valorise d'emblée un système «bien cadré », où tout serait écrit noir sur blanc, basé sur du « factuel » et non des impressions personnelles, qui donnerait « clairement» des indications au chef d'équipe et faciliterait en somme son travail d'évaluation des salariés et d'attribution des promotions. Toutefois, ce discours laisse ensuite la place à un positionnement plus mesuré.

Des effets de traduction par la maîtrise des objectifs du référentiel sont alors décelables. Le chef d'équipe peut utiliser différemment le référentiel selon l'interlocuteur qu'il a face à lui (Oiry et Sulzer, 2002); il peut par exemple fermer les yeux sur certains critères insatisfaisants pour remotiver et récompenser une personne méritante et possédant un bon état d'esprit :

\footnotetext{
«Y'a d'autres gens qui sont peut-être aussi incapables de réaliser certaines choses, mais qui sont méritants d'une promotion par leurs efforts, donc y'a aussi ce côté-là... [...] donc si je vois qu'un opérateur fait beaucoup d'efforts mais qu'il a beaucoup de mal à obtenir une classification supérieure, je fais en sorte de l'aider... C'est l'intention qui compte... » (Thierry, 37 ans, sans diplôme, chef d'équipe, ancien opérateur).
}

Pour les agents de maîtrise, la priorité quotidienne reste la gestion de la production même si celle-ci doit se faire au détriment de la gestion des carrières (Kalck, Marquette et Monchatre, 2002). En outre, la préservation de la paix sociale ou de la cohésion de l'équipe peut également influencer le processus d'évaluation (ibid., p. 150). Certains se voient ainsi accusés d'avoir été trop « généreux » dans l'attribution des coefficients, dans le seul but de sauvegarder de bonnes relations avec leurs subordonnés.

Enfin, il faut souligner que ces « dérives» (aux yeux des promoteurs de l'accord) sont rendues possibles par l'existence de marges d'interprétation dans les objectifs assignés par les référentiels de compétence. Si certains paraissent relativement clairs et précis, compte tenu notamment des outils de gestion disponibles (tableau de polyvalence, système informatique pour proposer une suggestion, etc.), l'« explicitation claire et univoque de compétences » est souvent rendue impossible par le caractère fondamentalement indexical du langage (Oiry et Sulzer, 2002, p. 33).

On peut alors supposer que le positionnement des chefs d'équipe face aux nouveaux accords va dépendre de leurs profils. Les plus âgés, souvent issus des rangs ouvriers, font généralement preuve d'une adhésion très limitée à la nouvelle conception du rôle du chef d'équipe : ces « gars du terrain» (pour reprendre leurs termes), qui 
voient de plus en plus de jeunes titulaires d'un BTS leur refuser la priorité pour les promotions au rang de chef d'atelier, critiquent férocement la surcharge de travail mais surtout un rôle centré sur la production de chiffres, de " beaux indicateurs ", de « beaux graphiques », stigmatisant les signes de distanciation avec le monde ouvrier (comme le port de la cravate ou l'usage de l'attaché-case), mais aussi l'éloignement physique de l'atelier pour se cantonner à un travail de « bureau ». Partageant l'ordre de l'ancienneté (quoique pour des raisons sensiblement différentes des managers de terrain évoqués plus haut), on pressent qu'ils participeront à leur mesure à une défense au moins partielle de ce principe.

\section{CONCLUSION}

Si la trajectoire scolaire prolongée des jeunes générations ouvrières est un facteur essentiel pour comprendre l'immense distance culturelle entre générations ouvrières et la difficulté à transmettre un héritage ouvrier à des jeunes générations partiellement acculturées à l'univers scolaire, refusant d'endosser une identité ouvrière et rêvant au contraire d'évasion hors du groupe ouvrier (Beaud et Pialoux, 1999), l'enquête permet ici de souligner comment les pratiques gestionnaires contribuent à travailler ces relations intergénérationnelles.

L'accord de classification étudié ici vient en effet renouveler une opposition des générations qui lui préexiste largement. Au cours des années 90, l'apparition des bacheliers professionnels modifie l'équilibre entre les critères de l'expérience et du diplôme pour l'évolution professionnelle des ouvriers. Or la définition de la « compétence » affichée dans le nouveau texte contient un déni de l'expérience qui vient fragiliser encore davantage les anciens. La défaite de l'ordre de l'ancienneté n'est pourtant pas actée, parce que ce principe reste partagé par les ouvriers professionnels jeunes et anciens, ainsi que par une partie de l'encadrement et des managers de proximité, et parce que la perte du coefficient lors d'une mobilité reste impossible. De fait, il ne s'agit pas là de la première attaque contre l'importance du facteur ancienneté dans les parcours professionnels. À l'instar du système de cotation de poste (Pitti, 2004), la grille de 1975 présentait initialement une absence de prise en compte de l'expérience. À travers l'application concrète, le facteur ancienneté avait cependant été largement réintroduit. On ne peut donc prédire si cette tentative sera plus effective que les précédentes (Reynaud, 2001). 
Refuser de tenir compte de

l'ancienneté comme facteur de qualification ouvrière, c'est plus fondamentalement remettre en cause le principe même d'une qualification ouvrière.
Quel est finalement l'enjeu crucial autour de cet ordre de l'ancienneté ? Dépassant la question d'un clivage intergénérationnel, nous défendons l'idée que refuser de tenir compte de l'ancienneté comme facteur de qualification ouvrière, c'est plus fondamentalement remettre en cause le principe même d'une qualification ouvrière. La logique compétence élaborée par la direction de l'entreprise peut être alors lue comme une tentative d'instrumentalisation des oppositions de générations matérialisées par les systèmes de gestion, afin de justifier la remise en cause des coefficients acquis au cours de la carrière: en vertu d'une «vérité des prix »érigée en principe de justice, qui consiste à récompenser les compétences réellement exercées à l'instant $\mathrm{T}$ sans tenir compte ni des capacités possédées dans l'absolu ni de celles démontrées par le passé, la déclassification potentielle d'un ouvrier professionnel dans la catégorie des opérateurs apparaît ainsi légitime aux yeux des promoteurs de l'accord. Dans un contexte de multiplication des situations de déclassements temporaires ou plus durables d'ouvriers professionnels sur des postes d'opérateurs (pour cause de ralentissement de l'activité des sites, de fermetures d'ateliers, de sureffectifs d'ouvriers professionnels notamment), la voie semble ouverte à une remise en cause de la qualification au cours de la carrière.

Les éléments présentés invitent donc à poursuivre l'investigation en suivant l'application et les réactions face à la « logique compétence » sur le moyen terme (tant des ouvriers professionnels que de l'encadrement de proximité), bref à dépasser les intentions décelées dans les nouveaux outils pour analyser les pratiques de classification (Lomba, Mazaud, 2004), pour observer la transformation des logiques initialement présentes et apprécier l'éventuelle capacité de résistance des différents groupes étudiés.

Séverine Misset Centre nantais de sociologie (CENS)

Université de Nantes severine.misset@univ-nantes.fr

\section{RÉFÉRENCES BIBLIOGRAPHIQUES}

Accord d'entreprise sur le développement et l'évolution professionnels des ouvriers de production, mars 2005.

Avenant à l'accord du 8 mars 2005 relatif au développement et à l'évolution professionnels des ouvriers de production, février 2007.

Accord national du 21 juillet 1975 sur la classification, modifié par les avenants du 30 janvier 1980, 21 avril 1981, 4 février 1983, 25 janvier 1990 et 10 juillet 1992, branche de la métallurgie. 
BEAUD S., 1996

BEAUD S., PIALOUX M., 1999

BRUCY G., 2007

CAMPINOS-DUBERNET M., 1995 « Baccalauréat professionnel : une innovation ? »,

CHARLOT B., FIGEAT M., 1985

CHATZIS K., de CONINCK F., ZARIFIAN Ph., 1995

COUSIN O., 2007

ECKERT H., 1999

ECKERT H., 2005

EYRAUD F., JOBERT A., ROZENBLATT P., TALLARD M., 1989

FLAMANT N., 2004

FLAMANT N., 2005

GRIGNON C., 1971

HALLIER P., THIESSET Ch., 1991

HATZFELD N., 2004

HUYEZ-LEVRAT G., 2007

KALCK P., MARQUETTE C., MONCHATRE S., 2002

LOMBA C., MAZAUD J-P., 2004 Formation Emploi, $\mathrm{n}^{\circ} 49$, p. 3-29.

« Les "bacs pro". La "désouvriérisation" du lycée professionnel », Actes de la recherche en sciences sociales, $\mathrm{n}^{\circ} 114$, p. $21-29$

Retour sur la condition ouvrière. Enquête aux usines Peugeot de Sochaux-Montbéliard, Paris : Fayard.

« De l'expérience reconnue à l'expérience déniée », Cahiers de la recherche sur l'éducation et les savoirs, nº 6, p. 273-298. Minerve.

"L'accord A. Cap 2000 : la "logique compétence" à l'épreuve des faits », Travail et Emploi, no 64, p. 35-47.

« La construction des inégalités hommes-femmes dans l'entreprise. Une analyse de cas dans la métallurgie », Sociologie du travail, vol. 49, n² 2, p. 195-219.

« L'émergence d'un ouvrier bachelier : les "bac pro" entre déclassement et recomposition de la catégorie des ouvriers qualifiés ", Revue française de sociologie, vol. 40, n², p. $227-253$

" "Déclassement" : de quoi parle-t-on? À propos des jeunes bacheliers professionnels, issus de spécialités industrielles... », Cereq Net.doc, n 19, p. 1-38.

Les Classifications dans l'entreprise, productions des hiérarchies professionnelles et salariales, Paris : La documentation française.

« Un introuvable conflit des générations », Futuribles, $n^{\circ} 299$, p. $65-67$

« Conflit de générations ou conflit d'organisation ? Un train peut en cacher un autre », Sociologie du Travail, vol. 47, n'2, p. 223-244.

L'Ordre des choses. Les fonctions sociales de l'enseignement technique, Paris : Minuit.

travail », Cereq Bref, n 69, p. 1-4.

« L'individualisation des carrières à l'épreuve. Les grippages de la mobilité sur les chaînes de Peugeot-Sochaux », Sociétés contemporaines, n 54 , p. 15-33

" "Jeunes vendeurs" contre "vieux techniciens", des compétences spécifiques ou des trajectoires divergentes? », Formation Emploi, nº 99, p. 47-59.

« L'histoire longue de la mise en œuvre d'un accord sur les compétences : quelques pistes d'analyse », in Brochier D. (coord.), La gestion des compétences : acteurs et pratiques, Paris : Economica, p. 137-161.

« Les classifications professionnelles en pratiques. Approches ethnographiques et historiques », Sociétés contemporaines, $\mathrm{n}^{\circ}$ 54, p. 7-11. 
MISSET S., EME B., HINAULT A.-C., 2004

MISSET S., 2009

MONCEL N., SULZER E., 2006

MONCHATRE S., 2002

MONCHATRE S., 2004

OIRY E., SULZER E., 2002

PEZET E., 2000

PITTI L., 2004

PROST A., 1981

REYNAUD J.-D., 1988

REYNAUD J.-D., 2001

RICHEBE N., 2002

SAGLIO J., 1988

SAGLIO J., 1991

TALLARD M., 2001

VENEAU P., MOUY Ph., 1995
Les processus identitaires d'entrée dans le travail ouvrier. Le cas des jeunes opérateurs à la chaîne, CRIDA-LISE/PSA Peugeot-Citroën/ANVIE, ronéo IRESCO-CNRS.

La déstabilisation des strates qualifiées du groupe ouvrier. Le cas des ouvriers professionnels de Peugeot-Citroën, Thèse de doctorat de sociologie, Université Paris Descartes.

« L'intégration des jeunes opérateurs chez PSA Peugeot Citroën. Contextes sociétaux, effets de sites et identités au travail », Notes Emploi Formation du Cereq, p. 1-65.

« Les avatars du modèle de la compétence. L'exemple d'un site de la sidérurgie », Formation Emploi, n 77, p. 51-68.

« De l'ouvrier à l'opérateur : chronique d'une conversion », Revue française de sociologie, vol. $45, n^{\circ} 1$, p. 69-102.

«Les référentiels de compétences : enjeux et formes », in BROCHIER D. (coord.), La Gestion des compétences : acteurs et pratiques, Paris : Economica, p. 29-47.

« Négociation collective et gouvernement des individus dans l'entreprise : la négociation des classifications dans la métallurgie (1968-1975) », Entreprises et histoire, n² 26, p. $74-88$.

« De la différenciation coloniale à la discrimination systémique ? La condition d'OS algérien à Renault, de la grille Parodi à la méthode Renault de qualification du travail (1945-1973) », La revue de l'IRES, n 46-3, p. 69-107.

Histoire générale de l'Enseignement et de l'Éducation en France, tome IV, « L'École et la Famille dans une société en mutation (1930-1980) », Nouvelle Librairie de France, Paris : G.V. Labat éditeur.

«Les régulations dans les organisations : régulation de contrôle et régulation autonome », Revue française de sociologie, vol. $29, n^{\circ} 1$, p. 19-33.

"Le management par les compétences : un essai d'analyse », Sociologie du travail, vol. 43, n 1, p. 7-31.

« Les réactions des salariés à la « logique compétence »: vers un renouveau de l'échange salarial ? », Revue française de sociologie, vol. 43, n 1, p. 99-126.

« Négociations de classifications et régulation salariale dans le système français de relations professionnelles », Travail et Emploi, n' 38 , p. 53-63.

« Les logiques de l'ordre salarial : avenants classification et salaires dans le système français de relations professionnelles », Formation Emploi, n 35, p. 35-46.

« L'introduction de la notion de compétence dans les grilles de classification : genèse et évolution », Sociétés contemporaines, $n^{\circ} 41-42$, p. 159-187.

« Des objectifs à la réalité. Les bacheliers professionnels industriels », Formation Emploi, n 49, p. 91-103. 